\title{
An ANOVA-Based GPS Multipath Detection Algorithm Using Multi-Channel Software Receivers
}

\author{
M.T. Brenneman, Y.T. Morton, and Q. Zhou \\ Dept. of Electrical and Computer Engineering \\ Miami University \\ Oxford, OH 45056
}

\begin{abstract}
We present a statistical detection test for GPS multipath based on the one-way ANOVA method. Given an antenna array with a GPS software receiver in tracking mode, the signal from each channel is correlated with a reference signal in blocks of one $\mathrm{CA}$ code period. When the relative phase delay for the direct GPS signal is stripped off from each channel, the expected values of the correlates is the same for all of the channels only if no multipath is present. A one-way ANOVA test can then used to determine if multipath is present.

An analysis of this method is presented which shows that the parameters affecting its detection performance can be grouped into three classes: the array size, the signal AOAs, and the processed multipath SNR. Receiver operating characteristic curves are given as a function of the processed multipath SNR for fixed array sizes. They show that good detection performance can be achieved under most operating conditions with less than $10 \mathrm{CA}$ code periods of data. It is also shown that the detection performance of this method improves as the multipath time delay decreases. This suggests this method could be a useful tool in aiding multipath mitigation techniques whose ability to detect multipath typically degrades as the multipath time delay decreases.
\end{abstract}

\section{INTRODUCTION}

Multipath is one of the major sources of error in precise position determination using GPS. A number of methods have been developed for multipath mitigation which can be grouped into two classes. The first class of techniques attempt to modify the receiver tracking loop in such a way that it is not affected by multipath. Methods that fall into this class include the narrow correlator [1], the strobe correlator [2], and Multipath Elimination Technology [3]. The second class of methods attempt to jointly estimate the direct and multipath signal parameters. They include the Multipath Estimating Delay Lock Loop (MEDLL) [4], modified RAKE delay lock loop [5], and Multipath Mitigation Technology [6].

The performance for both of classes of methods, however, is degraded when the relative time delay between the multipath and direct line of sight (LOS) GPS signal is short. A serious concern is that if the multipath is very close to the
LOS GPS signal in time, its presence and therefore the error it produces, could potentially go undetected. In the case of the first class of methods, for example, no detection is performed. In the multipath-estimator based methods, detection is implicitly incorporated into the method. This is because the number of multipath sources is one of the parameters being estimated. The estimation is often performed using the maximum likelihood (ML) method. It has been shown that the variance of Cramer-Rao lower bound (CRLB) for the ML estimated multipath parameters increases as the time-delay decreases [7], which could potentially lead to the misdetection of a single multipath source. Notwithstanding this problem, ML methods for multipath estimation are generally computationally expensive. This could be a limitation for receivers on a moving platform where prompt reporting on a rapidly changing multipath environment is required. Although recently improved versions of ML methods that reduce the complexity of the optimization process have been reported [8][9], a simpler and faster method for the detection of shorttime delay multipath would be desirable.

In this paper, we present a method that is designed to detect the presence of multipath signals by exploiting the spatial diversity between the direct GPS signal and its multipath. We shall show that our approach complements the previous methods, in that its detection performance is optimized exactly under those conditions where their performance is the worst.

The structure of this paper is as follows: Section II describes the mathematical model for the signals. Section III presents the theory and algorithm for multipath detection. The performance of this method is analyzed in section IV. Section $\mathrm{V}$ summarizes the results and highlights future improvements and directions.

\section{MATHEMATICAL SIGNAL MODEL}

Fig. 1 shows an idealized uniform linear array (ULA) configuration consisting of $\mathrm{K}$ elements spaced at one-half the nominal carrier wavelength. For a collection of isotropic array elements that are calibrated, the angle of arrival, denoted $\gamma$, determines the array's response. Under these conditions, the array's response, which is often called the steering vector and will be denoted by $\mathbf{S}$, is a complex vector with $\mathrm{K}$ components having the parametric form: 


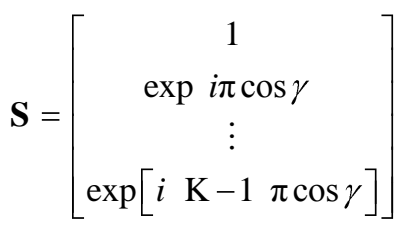

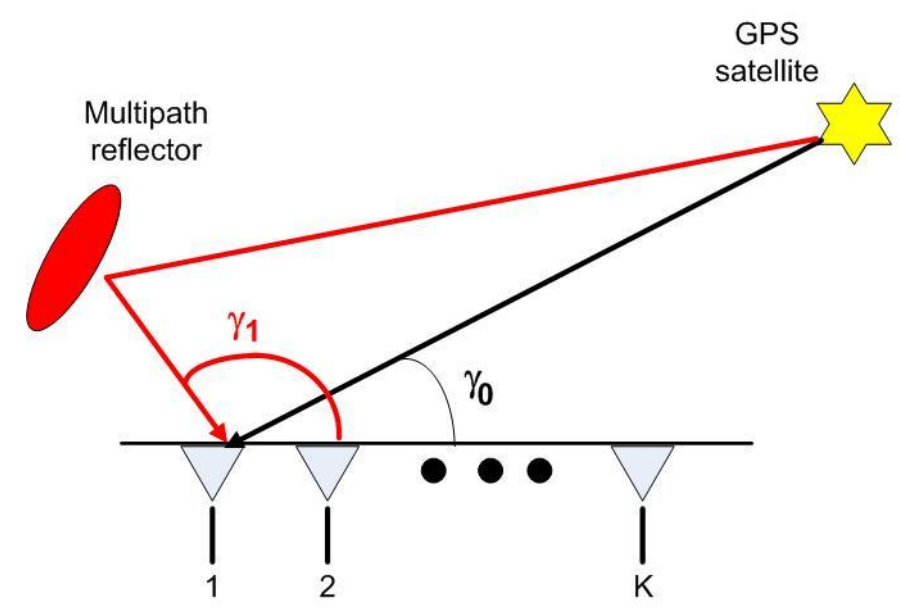

Figure 1. Simplified Model System

An ideal ULA of K elements collects the signals from the direct GPS and its multipath signals. A key property for each signal is the orientation of its wave vector relative to the array axis, known as its angle of arrival and denoted by $\gamma$.

In a multipath environment, the signal is typically modeled as the sum of a desired GPS signal, M multipath signals, and random channel noise. All of the deterministic signals will be considered stationary over the time interval of observation. The noise from each channel is distributed as $\operatorname{CWGN}\left(0, \sigma^{2}\right)$ and is uncorrelated both spatially and temporally. The mathematical model for the signal used will be its complex, digitized form at baseband sampled with frequency $f_{s}$. Neglecting the navigation data bit, it can be expressed in the form:

$$
\mathbf{X} \mathrm{t}_{\mathrm{n}}=\sum_{\mathrm{j}=0}^{\mathrm{M}} \mathrm{A}_{\mathrm{j}} \mathrm{C} \mathrm{t}_{\mathrm{n}}-\tau_{\mathrm{j}} \exp \left[i \omega \mathrm{t}_{\mathrm{n}}+\psi_{\mathrm{j}}\right] \mathbf{S}_{\mathrm{j}}+\boldsymbol{\varepsilon} \mathrm{t}_{\mathrm{n}}
$$

where $\mathrm{n}$ represent the sample index, $j=0$ corresponds to the direct LOS GPS signal, and

$$
\begin{aligned}
\mathrm{C} \mathrm{t} & =\text { GPS CA code for desired signal } \\
\mathrm{A}_{\mathrm{j}} & =\text { Signal amplitude } \\
\tau_{\mathrm{j}} & =\text { Relative time delay to LOS signal } \\
\psi_{\mathrm{j}} & =\text { Relative carrier phase to LOS signal } \\
\omega & =\text { Intermediate frequency with Doppler shift } \\
\mathbf{S}_{\mathrm{j}} & =\text { Steering vector }
\end{aligned}
$$

Some additional notation that will be used throughout this paper is as follows: vectors will be denoted by boldface type, conjugate transposes by superscript $\mathrm{H}$, transposes by superscript $\mathrm{T}$, and conjugates by a superscript $*$. The expected value of a random variable will be denoted as $\mathrm{E}\{\}$.

\section{METHODOLOGY}

The method we propose is a binary detection method for multipath cast in the form of a simple statistical hypothesis test. The null hypothesis is chosen to be the condition that no multipath is present and the alternative hypothesis is the condition that multipath is present. In the first sub-section, we quickly review the analysis of variance or ANOVA method used in our method to test these two hypotheses. In the second section, we motivate and explain the signal processing steps necessary to prepare the signal so it can be tested with ANOVA. We end this section with a discussion on how the operating conditions which will satisfy a given level of statistical performance are determined.

\section{A. ANOVA}

Analysis of Variance or ANOVA is a standard statistical method to test whether the mean of a random variable is the same in multiple populations [10]. ANOVA starts with K distinct populations each containing random samples of a given random variable $Z$. In our analysis, we will use balanced ANOVA, in which all of the populations contain the same number of samples. Letting $\mathrm{n}_{\mathrm{K}}$ be the number of samples in each population and $Z_{h, k}$ denote the $h^{\text {th }}$ sample from the $\mathrm{k}^{\text {th }}$ population, if the following conditions are satisfied for all $h, h^{\prime}=1, \ldots, \mathrm{n}_{\mathrm{K}}$ and $\mathrm{k}, \mathrm{k}^{\prime}=1, \ldots, \mathrm{K}$ :

$$
\begin{aligned}
& \mathrm{Z}_{\mathrm{h}, \mathrm{k}} \stackrel{\text { i.i.d. }}{\sim} \mathrm{N} \mu_{\mathrm{k}}, \sigma_{\mathrm{k}}^{2} \\
& \sigma_{\mathrm{k}}^{2}=\sigma^{2} \\
& \text { Covar } \mathrm{Z}_{\mathrm{h}, \mathrm{k}} \mathrm{Z}_{\tilde{h}, \tilde{\mathrm{k}}}=\sigma^{2} \delta_{\mathrm{h}, \tilde{\mathrm{h}}} \delta_{\mathrm{k}, \tilde{\mathrm{k}}}
\end{aligned}
$$

then ANOVA can be used to test the following statistical hypotheses:

$$
\begin{array}{ll}
\mathrm{H}_{0}: \mu_{\mathrm{k}}=\mu_{\mathrm{j}} & \text { for all } \mathrm{k}, \mathrm{j}=1, \ldots, \mathrm{K} \\
\mathrm{H}_{1}: \mu_{\mathrm{k}} \neq \mu_{\mathrm{j}} & \text { for some } \mathrm{k}, \mathrm{j}=1, \ldots, \mathrm{K}
\end{array}
$$

ANOVA is based on a comparison of two sample variances. The first variance, called the mean-square error within populations and denoted by $\mathrm{MSE}_{\mathrm{w}}$, estimates the average sample variance taken over all the populations:

$$
\operatorname{MSE}_{w}=\frac{\sum_{k=1}^{K} \sum_{j=1}^{n_{k}} Z_{j, k}-\hat{\mu}_{k}{ }^{2}}{\mathrm{~K} \mathrm{n}_{K}-1}
$$


Because the second condition in (4) requires the variance of the random variables to be the same for all of the populations, the $\mathrm{MSE}_{\mathrm{w}}$ is an unbiased estimator for the true sample variance.

The second variance is the variance between the sample means. It is known as the mean-square error between groups and is given by:

$$
\mathrm{MSE}_{\mathrm{b}}=\frac{\sum_{\mathrm{k}=1}^{\mathrm{K}} \mathrm{n}_{\mathrm{K}} \hat{\mu}_{\mathrm{k}}-\bar{\mu}^{2}}{\mathrm{~K}-1}
$$

where $\bar{\mu}$ is the average of the $\mathrm{K}$ populations means.

Under $\mathrm{H}_{0}$, it can be shown that $\mathrm{MSE}_{\mathrm{b}}$ is also an estimate for the true sample variance, and by Cochran's theorem, it can be shown that $\mathrm{MSE}_{\mathrm{b}}$ and $\mathrm{MSE}_{\mathrm{w}}$ are independent chisquare random variables having degrees of freedom $(\mathrm{K}-1)$ and $\mathrm{K}\left(\mathrm{n}_{\mathrm{K}}-1\right)$, respectively. Hence their ratio follows a central $\mathrm{F}$ distribution with $(\mathrm{K}-1)$ numerator degrees of freedom and $\mathrm{K}\left(\mathrm{n}_{\mathrm{K}}-1\right)$ denominator degrees of freedom. Under $\mathrm{H}_{1}$, even though $\mathrm{MSE}_{\mathrm{w}}$ and $\mathrm{MSE}_{\mathrm{b}}$ are still independent, the fact that $\mathrm{MSE}_{\mathrm{b}}$ is a non-central chi-square random variable makes their ratio a non-central $F$ distribution. Letting $\mathrm{F}\left(\alpha \mid(\mathrm{K}-1), \mathrm{K}\left(\mathrm{n}_{\mathrm{K}}-1\right)\right)$ denote the critical value for the hypothesis test having size $\alpha$, the decision rule in ANOVA is:

$$
\begin{aligned}
& \frac{\mathrm{MSE}_{\mathrm{b}}}{\mathrm{MSE}_{\mathrm{w}}}>\mathrm{F} \alpha \mid \mathrm{K}-1, \mathrm{~K} \mathrm{n} \mathrm{K}_{\mathrm{K}}-1 \Rightarrow{\text { Reject } \mathrm{H}_{0}} \\
& \frac{\mathrm{MSE}_{\mathrm{b}}}{\mathrm{MSE}_{\mathrm{w}}} \leq \mathrm{F} \alpha \mid \mathrm{K}-1, \mathrm{~K} \mathrm{n} \mathrm{K}_{\mathrm{K}}-1 \Rightarrow \text { Fail to reject } \mathrm{H}_{0}
\end{aligned}
$$

\section{B. Algorithm for Multipath Detection with ANOVA}

In the context of multipath detection, we use ANOVA to determine if a signal contains only the direct GPS signal, or if it also contains its multipath signals. Based on the signal model in (2), the statistical test we wish to perform can be stated as:

$$
\begin{array}{ll}
H_{0}: M=0 & \text { i.e. no multipath present } \\
H_{0}: M>0 & \text { i.e. multipath is present }
\end{array}
$$

To aid in explaining how ANOVA can be used to perform this test, we will introduce the two signals for a $\mathrm{K}$ element ULA, $\mathbf{X}_{\mathbf{0}}$ and $\mathbf{X}_{\mathbf{1}}$ :

$$
\begin{aligned}
& \mathbf{X}_{0} \mathrm{t}_{\mathrm{n}}=\mathrm{A}_{0} \mathrm{C} \mathrm{t}_{\mathrm{n}} \exp i \omega \mathrm{t}_{\mathrm{n}} \mathbf{S}_{\mathbf{0}}+\boldsymbol{\varepsilon} \mathrm{t}_{\mathrm{n}} \\
& \mathbf{X}_{1} \mathrm{t}_{\mathrm{n}}=\sum_{\mathrm{j}=0}^{\mathrm{M}} \mathrm{A}_{\mathrm{j}} \mathrm{C} \mathrm{t}_{\mathrm{n}}-\tau_{\mathrm{j}} \exp \left[i \omega \mathrm{t}_{\mathrm{n}}+\psi_{\mathrm{j}}\right] \mathbf{S}_{\mathrm{j}}+\boldsymbol{\varepsilon} \mathrm{t}_{\mathrm{n}}
\end{aligned}
$$

where $n=1, \ldots \mathrm{N}$ (total number of samples) and $\mathrm{M}>0$. We will refer to these two signals as test signals because they represent the signals under the two hypotheses we wish to test.

No method for GPS signal detection can be applied directly to the input signal. This is due to the fact that the GPS signal is by construction a weak direct spreadspectrum signal which is not statistically detectable. Assuming that the receiver is already in tracking mode, we can correlate the signal from each channel with an estimate for the reference signal of the direct GPS signal in order to increase its effective signal to noise ratio. Using our previous notation, the discrete estimated reference signal at time $t_{n}$ can be written in the form:

$$
\hat{\mathrm{r}} \mathrm{t}_{\mathrm{n}}=\mathrm{C} \mathrm{t}_{\mathrm{n}}-\delta_{\tau} \exp \left[\mathrm{i} \hat{\omega} \mathrm{t}_{\mathrm{n}}+\hat{\psi}_{0}\right]
$$

where $\delta_{\tau}$ is the error of the code phase tracking loop.

$\mathrm{n}_{\mathrm{K}}$ correlations will be performed using consecutive blocks of data, each having a length of one CA code period $\left(\mathrm{T}_{\mathrm{CA}}=1 \mathrm{msec}\right)$. It is not coincidental that $\mathrm{n}_{\mathrm{K}}$ was also used in the last sub-section to denote the number of data points in each population for a balanced ANOVA experiment. As we will see later, each $\mathrm{CA}$ code period will contribute a single data point to the populations in our ANOVA analysis. To insure that we have enough data points for reliable statistical inference, while also minimizing the computation time required for our analysis, the number of $\mathrm{CA}$ code periods is typically chosen to be between 3 and 10 (i.e. $3 \leq$ $\left.\mathrm{n}_{\mathrm{K}} \leq 10\right)$.

The signal is correlated over each successive CA code period with the estimated reference signal given in (11). In the correlations, the difference between the estimated and true carrier frequency will be approximated to be zero. For a GPS receiver in tracking mode, the typical error in the frequencies is a few Herz, and since the total integration time is at most $10 \mathrm{msec}$, this approximation is valid. Under this condition, the correlation for the $\mathrm{j}^{\text {th }}$ source from each time block will be the same, and is given by [11]:

$$
\rho_{\mathrm{j}}=\mathrm{A}_{\mathrm{j}} \exp \left[i \psi_{\mathrm{j}}-\hat{\psi}_{0}\right]\left(1-\frac{\left|\tau_{\mathrm{j}}-\delta_{\tau}\right|}{\mathrm{T}_{\mathrm{CA}}}\right)
$$

Both test signals in (10) have the same noise, so we can evaluate the noise contribution to the correlator output from the $i^{\text {th }} \mathrm{CA}$ code period. If we define:

$\beta \mathrm{n}=-\hat{\psi}_{0}+\pi\left(\frac{1-\operatorname{Sign} \mathrm{C} \mathrm{t}_{\mathrm{n}}-\delta_{\tau}}{2}\right)$

then the $\mathrm{k}^{\text {th }}$ component of the post-correlated noise from the $i^{\text {th }} \mathrm{CA}$ code period can be expressed as: 


$$
\varepsilon_{\mathrm{k}}^{\prime} \mathrm{i}=\frac{\sum_{\mathrm{n}=1+\mathrm{i}-1 *^{*} \mathrm{~N}_{\mathrm{ca}}}^{\mathrm{i} \mathrm{N}_{\mathrm{ca}}} \exp [i \beta \mathrm{n}] \varepsilon_{\mathrm{k}} \mathrm{t}_{\mathrm{n}}}{\mathrm{N}_{\mathrm{CA}}}
$$

where $\mathrm{N}_{\mathrm{CA}}$ denotes the total number of data points sampled in one CA code period.

Since each component of the random channel noise at each time sample is modeled as a zero-mean complex normal random variable, multiplication of each element by a phase factor changes neither the distribution nor covariance properties of the noise. From this fact, it directly follows that:

$$
\varepsilon_{\mathrm{k}}^{\prime} \mathrm{i} \stackrel{\text { i.i.i. }}{\sim} \mathrm{CWGN}\left(0, \frac{\sigma^{2}}{\mathrm{~N}_{\mathrm{CA}}}\right)
$$

which holds for all $\mathrm{k}=1, \ldots, \mathrm{K}$ and all $\mathrm{i}=1, \ldots, \mathrm{n}_{\mathrm{K}}$. The condition that the post-correlated noise samples are independent follows from the third condition in (4).

Combining the correlations of the deterministic and noise signals, the total output signal for the two test signals from the $\mathrm{i}^{\text {th }} \mathrm{CA}$ code period can be written as:

$$
\begin{aligned}
& \mathbf{Y}_{\mathbf{0}} \mathrm{i}=\rho_{0} \mathbf{S}_{\mathbf{0}}+\boldsymbol{\varepsilon}^{\prime} \mathrm{i} \\
& \mathbf{Y}_{\mathbf{1}} \mathrm{i}=\sum_{\mathrm{j}=0}^{\mathrm{M}} \rho_{\mathrm{j}} \mathbf{S}_{\mathrm{j}}+\boldsymbol{\varepsilon}^{\prime} \mathrm{i}
\end{aligned}
$$

ANOVA can be used when K populations of normally distributed random variables are independent and have the same variance. From (15), it follows that our signal model under both hypotheses satisfies the assumptions necessary to use ANOVA. However, for an ANOVA test to be successful, the data must also satisfy the condition that the mean of each population will be the same when $\mathrm{H}_{0}$ holds. If we consider each of the channels as being a population (so our K populations are represented by the $\mathrm{K}$ channels), we see from (16) that the expected values of our $\mathrm{K}$ "populations" under $\mathrm{H}_{0}$ are:

$$
\mathrm{E} \mathbf{Y}_{0} \mathrm{i}=\rho_{0}\left[\begin{array}{c}
1 \\
\exp \left[i \pi \cos \gamma_{0}\right] \\
\vdots \\
\exp \left[i \mathrm{~K}-1 \pi \cos \gamma_{0}\right]
\end{array}\right]
$$

Equation (17) shows us that under $\mathrm{H}_{0}$ the means for the different channels are generally not all equal. To make them equal under $\mathrm{H}_{0}$, the $\mathrm{k}^{\text {th }}$ channel signal needs to be multiplied by the weight:

$$
\mathrm{w}_{\mathrm{k}}=\exp \left[-i \mathrm{k}-1 \pi \cos \gamma_{0}\right]
$$

which can be constructed, assuming that the direct GPS signal's AOA is known. After the application of the weight, the test signals (which we'll denote by $\mathrm{Z}$ ) for the $\mathrm{i}^{\text {th }} \mathrm{CA}$ code period have the form:

$$
\begin{aligned}
& \mathbf{Z}_{\mathbf{0}}=\rho_{0}\left[\begin{array}{c}
1 \\
\vdots \\
1
\end{array}\right]+\boldsymbol{\varepsilon}^{\prime \prime} \mathrm{i} \\
& \mathbf{Z}_{\mathbf{1}}=\rho_{0}\left[\begin{array}{c}
1 \\
\vdots \\
1
\end{array}\right]+\sum_{\mathrm{j}=1}^{\mathrm{M}} \rho_{\mathrm{j}}\left[\begin{array}{c}
1 \\
\exp \left[i \Delta_{\mathrm{j}}\right.
\end{array}\right] \\
& \vdots \\
& \exp \left[\begin{array}{lll}
i & \mathrm{~K}-1 & \Delta_{\mathrm{j}}
\end{array}\right]+\boldsymbol{\varepsilon}^{\prime \prime} \mathrm{i}
\end{aligned}
$$

where $\Delta_{\mathrm{j}}=\pi\left[\cos \left(\gamma_{\mathrm{j}}-\cos \gamma_{0}\right)\right]$. Since the weight is a phase factor, $\varepsilon^{\prime \prime}(\mathrm{i})$ has the same distribution as $\varepsilon^{\prime}(\mathrm{i})$.

From (19) it follows that our signal now not only satisfies the assumptions necessary to use ANOVA, but that it can be used with ANOVA to perform the statistical hypothesis test in (9). From the first line of (19), it follows that the expected values for the final output signals from each channel are the same under $\mathrm{H}_{0}$. Under the alternative hypothesis however, the second line of (19) shows that expected value of the final output signal will be different for each channel due to the contribution of the multipath signals. The simplified block diagram shown in Fig. 2 summarizes the overall algorithm used to detect multipath with ANOVA for a K element antenna array.

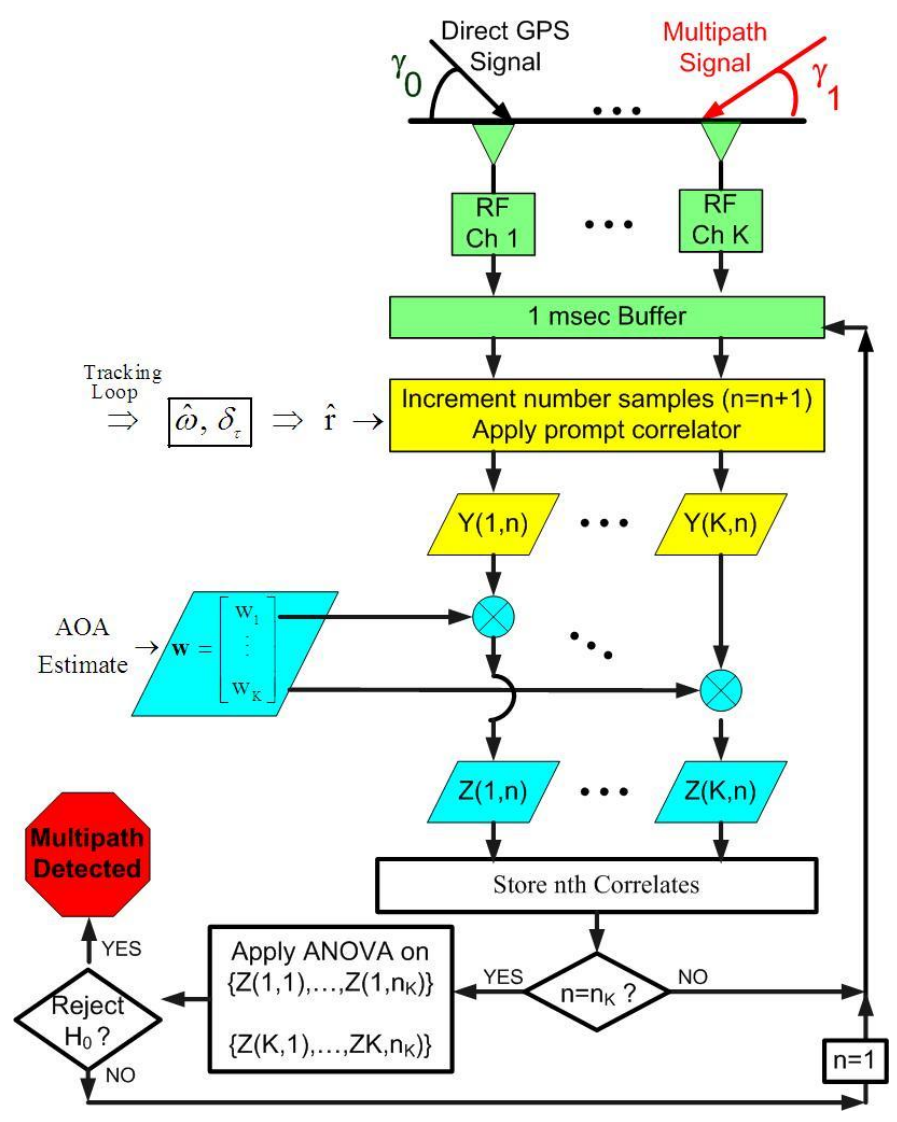


Fig. 2. Flowchart for ANOVA with GPS Software Receiver

\section{ANOVA Experimental Design}

The algorithm just described omits an important preliminary step which is central to all ANOVA experiments: sample size determination. In this section, we will consider this simple design issue and show how the problem of sample size determination is solved for multipath detection.

In signal detection problems, we want to choose our system parameters so that our detection method will perform according to some pre-determined performance conditions. The two measures of detection performance are the false alarm rate $\alpha$ and the missed detection probability $\beta$. One typically sets predetermined tolerance levels for both $\alpha$ and $\beta$ that we want the detection method to satisfy.

Sample size is an important parameter that affects both $\alpha$ and $\beta$. Although sample size determination for ANOVA is in general rather complicated, we can nevertheless illustrate the basic underlying concept of how it works. Fig. 3a shows a hypothetical probability density for the sampling distribution of the mean in a single population under $\mathrm{H}_{0}$ and $\mathrm{H}_{1}$ based on some fixed sample size. The predetermined condition on $\alpha$ determines the critical value, $\mathrm{Z}_{\mathrm{C}}$, upon which the decision rule is based:

$$
\begin{aligned}
& \mathrm{Z}>\mathrm{Z}_{\mathrm{C}} \Rightarrow \text { Reject } \mathrm{H}_{0} \\
& \mathrm{Z} \leq \mathrm{Z}_{\mathrm{C}} \Rightarrow \text { Fail to reject } \mathrm{H}_{0}
\end{aligned}
$$

Based on this decision rule, it follows that $\beta$ is the probability that the mean will be less than $Z_{C}$ when $H_{1}$ holds. We see from Fig. 3a that this is the shaded area under the probability density for $\mathrm{H}_{1}$ to the left of $\mathrm{Z}_{\mathrm{C}}$. Although the decision rule insures that the false alarm rate is satisfied, what if the area under the curve is greater than the value of $\beta$ desired? The solution to this problem lies in the fact that the variance of both distributions in ANOVA is inversely proportional to the sample size. Thus, increasing the sample size makes both distributions more concentrated about their means and reduces the probability density in the tails of the distribution. Fig. $3 b$ shows the same distribution after the sample size has been increased by a factor of four. We see quite clearly that $\beta$, the probability the mean will fall below $\mathrm{Z}_{\mathrm{C}}$ when $\mathrm{H}_{1}$ is true is substantially decreased.
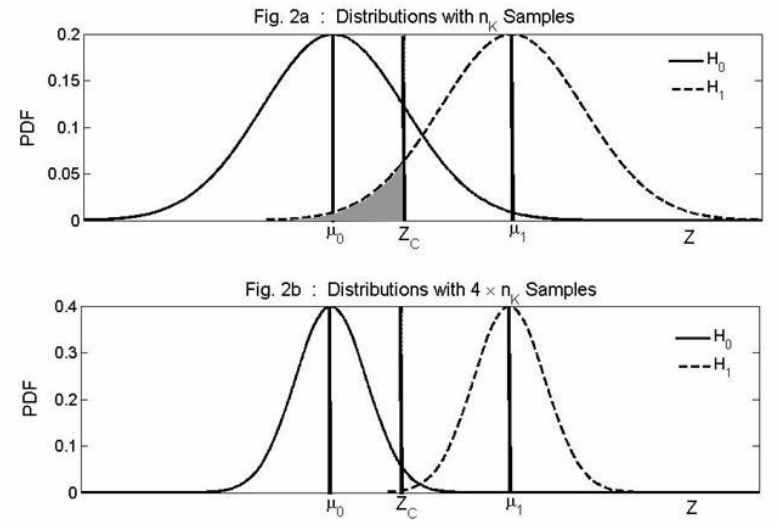

Fig. 3

If we continue to increase the sample size, the probability of both $\alpha$ and $\beta$ would go to zero. While that may seem very desirable, in practice it is often more useful to try to collect as few samples as possible. In the case of multipath detection for example, collecting more samples requires longer times. In an environment with a rapidly changing multipath environment, prompt reporting of the multipath conditions is an important consideration. The goal then is to find the minimum sample size that will satisfy both $\alpha$ and $\beta$.

There are a variety of statistical methods designed for determining the sample size with ANOVA [12]. To implement such methods however, one needs an estimate of how close the mean values of the statistic under $\mathrm{H}_{0}$ and $\mathrm{H}_{1}$ are. Referring to Fig. 3, one could imagine that as the means of the two distributions approached one another (while the variance of the distributions remained the same), the number of samples required to make the distributions sufficiently well separated would have to increase. Therefore, to determine the sample size for a given performance specification, an estimate of the minimum difference between $\mu_{0}$ and $\mu_{1}$ one wishes to detect, must be known a priori.

For the problem of multipath detection, an estimate of the minimum difference in the sample means is difficult to determine. For that reason, we instead use Monte-Carlo simulations to estimate the sample size required to meet a specified performance criteria. The parameters in our simulations are those which affect detection performance: the number of array elements $\mathrm{K}$, the data length, the direct signal AOA, and the multipath signal parameters including the AOA, relative time delay, and signal strength. Based on our analysis, we can define a parameter $\Gamma$ which essentially represents the effective multipath signal strength at the output of our correlation and weighting algorithm:

$$
\Gamma=10 \log _{10}\left[\sqrt{\mathrm{N}_{\mathrm{CA}} \mathrm{n}_{\mathrm{K}}} 10^{\mathrm{SNR} / 20}\left(1-\frac{\left|\tau_{1}\right|}{\mathrm{T}_{\mathrm{CA}}}\right)\right]
$$


To obtain a quantitative understanding of the dependence $\Gamma$ has on its parameters, Fig. 4 shows a contour plot of $\Gamma$ as a function of the multipath input SNR and time delay. The third parameter on which $\Gamma$ depends, total number of data points $\left(\mathrm{N}=\mathrm{n}_{\mathrm{K}} \mathrm{N}_{\mathrm{CA}}\right)$ was kept fixed at 25,000 (5 CA code periods sampled at a rate of $5 \mathrm{MHz}$ ).

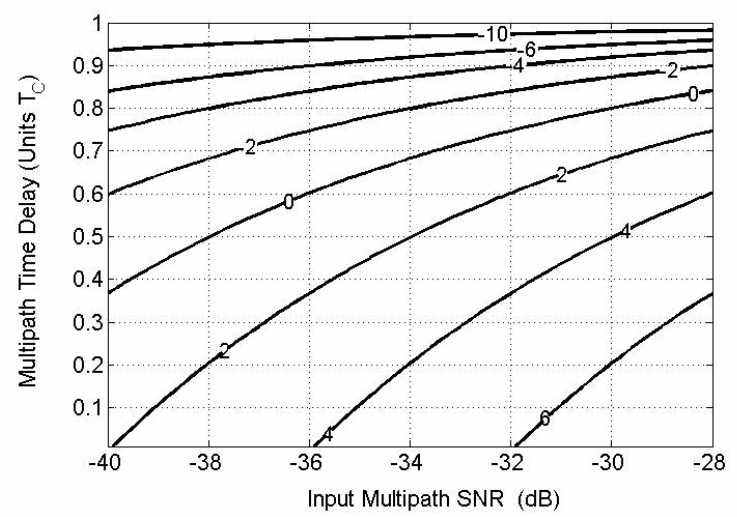

Fig.4 Effect of Multipath Parameters on Net Multipath SNR Using (21) with $\mathrm{N}=25,000, \Gamma$ is plotted as a function of the multipath parameters. Contour plot values are in units of $\mathrm{dB}$.

For multipath whose AOA is well separated from that of its direct signal, $\Gamma$ principally determines the detection algorithm's performance for a fixed array size. This can be demonstrated by the simulation results shown in Fig. 5 where both $\Gamma$ and the mean $\mathrm{F}$ statistic values are plotted as contours functions of the number of CA code periods $n_{K}$, and the multipath SNR. The simulation is performed with a signal model containing a direct signal, a single multipath, and noise received by a three element ULA with $\tau_{1}$ fixed at $0.2 * \mathrm{~T}_{\mathrm{CA}}$. The sampling frequency is again $5 \mathrm{MHz}$ and the multipath and direct signal AOAs are $45^{\circ}$ and $86^{\circ}$ respectively. For each pair of multipath SNR and number of CA codes sampled, 2,500 Monte-Carlo simulations were performed and the average F-statistic value computed.

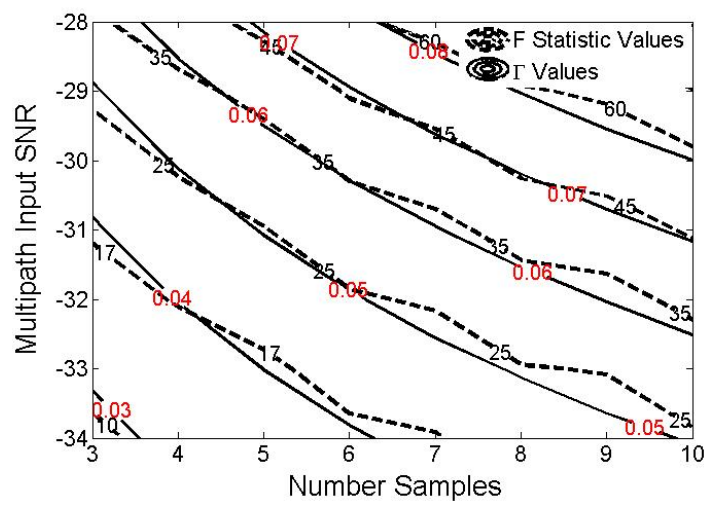

Fig. 5 Correlation between $\Gamma$ and the Detection Statistic The F-statistic values estimated from 2,500 Monte Carlo simulations show the same functional trend as the $\Gamma$ values.
We see that the contours of $\Gamma$ and the F-statistic correlate very well, indicating that the detection performance can be completely accounted for by $\Gamma$. This agreement is intuitively sound because the net multipath SNR efectively specifies the smallest significant signal that can be detected.

Based on this result, it is possible to determine the sample size for a given pair of $(\alpha, \beta)$. Fig. 6 is a contour plot of $\beta$ for a three element ULA using simulation. The $\beta$ values are estimated from 5000 Monte-Carlo simulations for given pairs of $\alpha$ and $\Gamma$ values, by determining the percentage of $F$ statistic values that fell below the critical value for $\alpha$. We see from the plot for example, that for the multipath detection to have a false alarm rate of no more than 0.05 and a missed detection probability of no greater than 0.05 , a minimum value of $\Gamma=3.266 \mathrm{~dB}$ would be required. If the minimum multipath SNR to be detected is specified along with the maximum time delay and sampling rate, it is possible, using equation (21) to compute the minimum number of samples required. For example, substituting the values $\Gamma=3.3 \mathrm{~dB}$, along with a multipath SNR of $-34 \mathrm{~dB}$, a sampling rate of $5 \mathrm{MHz}$, and the maximum time delay of $0.5 \mathrm{~T}_{\mathrm{CA}}$ into equation (21), we find that a sample size of 9 , or 9 CA code periods, would be required.

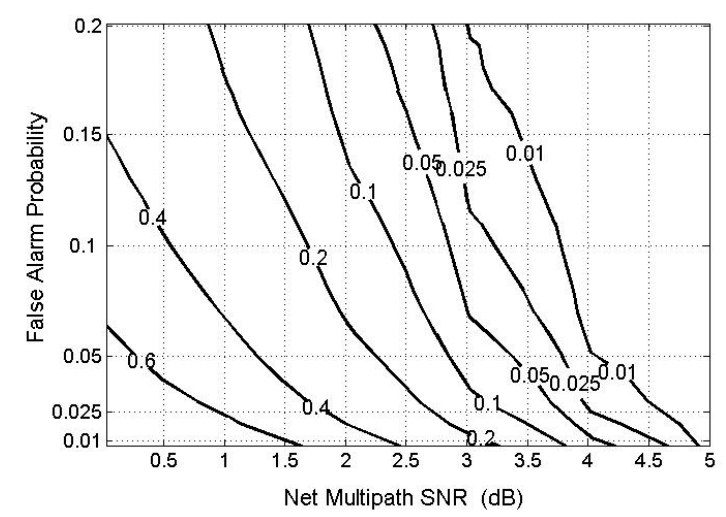

Fig. 6 Determination of sample size for 3 element ULA The contour for the desired value of $\beta$ is found. The point where this contour intersects the desired value of $\alpha$ is then found. The value of $\Gamma$ at which they intersect can then be used with (21) to determine the sample size.

\section{RESULTS}

We will present results fromMat $\mathrm{LAB}$ simulations to evaluate the detection performance of ANOVA. The relevant parameters on which the performance depends are $\Gamma$ (which contains the total number of CA codes used along with the multipath SNR and time delay), the number of array elements, and the AOAs of multipath and direct GPS signal.

Fig. 7 illustrates how the detection performance is affected by $\Gamma$ and $K$, the number of array elements. Two sets of levels curves for $\Gamma$ are plotted as a function of the false 
alarm rate and missed detection rate. The first group corresponds to an array with 3 elements, while the second group is for an array with 7 elements.

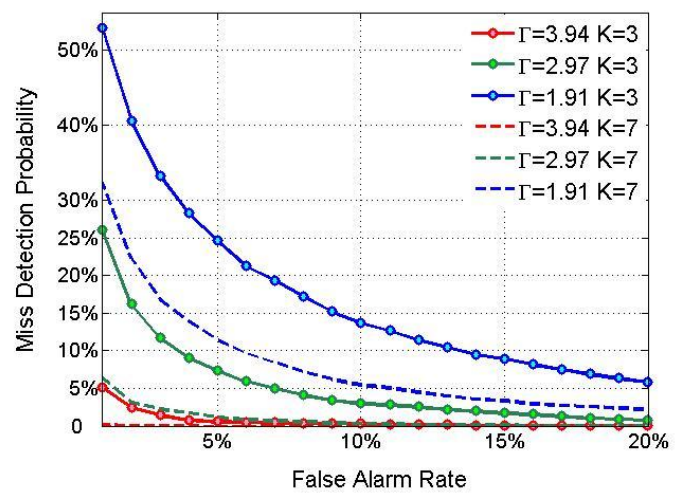

Fig. 7. Performance Curves for Single Multipath Signal The relationship between $\alpha, \beta$, and $\Gamma$ for a 3 and 7 element array is shown. Given any 2 of these 3 quantities, the third can be estimated from the plot.

Fig. 7 shows that for a fixed array dimension, a larger $\Gamma$ value corresponds to lower miss detection rate and false alarm rate. This is intuitively expected since a larger $\Gamma$ value represents stronger processed multipath signals. For the same $\Gamma$ value, a larger array also reduces the false alarm and miss detection rate. For example, if acceptable false alarm and miss detection rates of $5 \%$ and $7.5 \%$ respectively are chosen, then the multipath that can meet this criterion should have a minimum $\Gamma$ value of 2.97 if the array has 3 elements. For a 7 element array, the corresponding $\Gamma$ value is around 2.5.

What are the multipath signal parameters for the above mentioned $\Gamma$ values? Similar to Fig. $4, \Gamma$ is plotted as a function of the multipath parameters, but now two sets of contours for $\Gamma$ are shown. The first set of contours are the $\Gamma$ values which would be obtained using the lower bound for the number of samples, $n_{K}=3$. The second set of contours are the $\Gamma$ values obtained using the upper bound, $\mathrm{n}_{\mathrm{K}}=10$. Fig. 8 provides a quantitative description of the $\Gamma$ value's dependency on the basic multipath signal parameters, $\tau_{1}$ and SNR. Two sets of curves are plotted in Fig. 8. The solid lines are generated for $n_{k}=10 C A$ code periods and the dashed lines are for $3 \mathrm{CA}$ code periods. Based on this figure, we see that for $\Gamma=2.97$, the multipath SNR has to be larger than $-41 \mathrm{~dB}$ in order to meet the detection criteria, if $\mathrm{n}_{\mathrm{k}}=10$. For $\mathrm{n}_{\mathrm{k}}=3$, the minimum multipath SNR is $-35.7 \mathrm{~dB}$. For a given multipath SNR, the $\Gamma$ value sets the upper limit of the multipath delay time that can meet the previously stated detection criteria. For example, if the multipath SNR is $-35 \mathrm{~dB}$, then the maximum multipath delays are $0.5 \mathrm{~T}_{\mathrm{CA}}$ and $0.1 \mathrm{~T}_{\mathrm{CA}}$ for $\mathrm{n}_{\mathrm{k}}=10$ and 3 respectively.

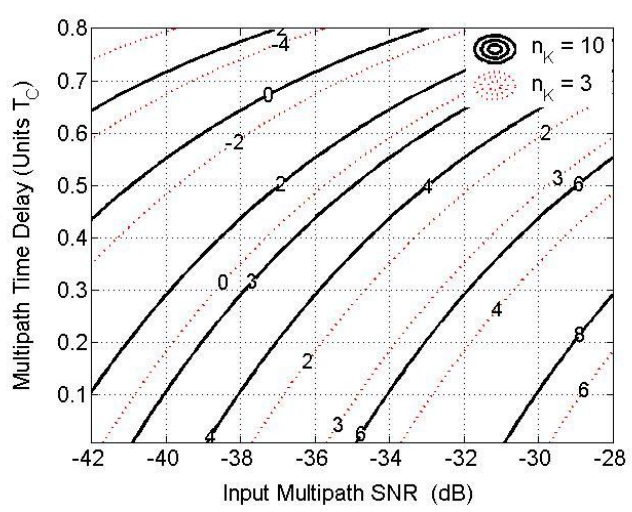

Fig. 8 Dependence of $G$ on multipath time delay, the input multipath SNR and number of samples collected

Fig. 8 shows that if all of the system parameters are fixed except the multipath time delay, $\Gamma$ increases as the time delay decreases. From Fig. 7, it was seen that both detection errors decrease as $\Gamma$ increases, hence we can conclude that there is a uniform improvement in detection performance as the multipath time delay decreases. This is an interesting result because it is the exact opposite of typical MLE methods such as the MEDLL, whose performance becomes worse as the time delay decreases. The basis for this result lies in the fact that the ANOVA method makes use of differences in the AOAs between the direct and multipath signal. By using the signal's spatial, rather than its temporal diversity, ANOVA does not encounter this limitation due to the time delay.

Fig. 9 shows how significant the improvement in detection is as the multipath time delay decreases. Using a desired direct GPS signal with an input SNR of $-18 \mathrm{~dB}$ and a multipath signal with an input SNR of $-28 \mathrm{~dB}$, the time delay is changed in increments of $0.1 * \mathrm{~T}_{\mathrm{CA}}$. For each time delay, 5,000 Monte-Carlo simulations are run and the critical value is chosen at which $\alpha=\beta$. We see that at a time delay of $0.7^{*} \mathrm{~T}_{\mathrm{CA}}$, a probability of false alarm and missed detection of 0.2 can be achieved, but at a $0.3^{*} \mathrm{~T}_{\mathrm{CA}}$, the probability of false alarm and missed detection drop dramatically to less than 0.01 .

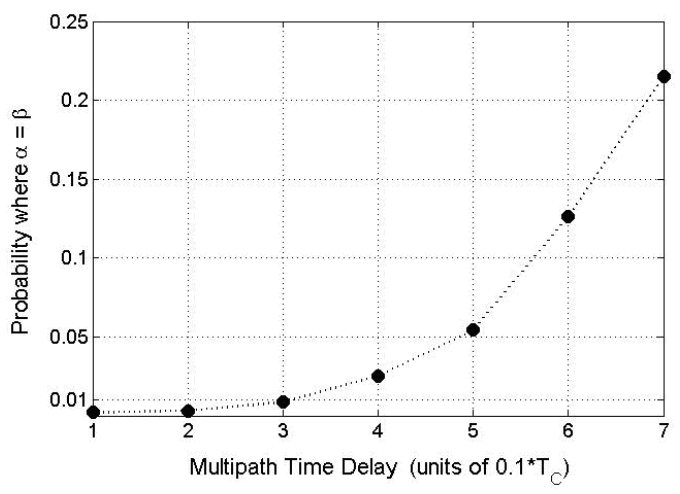

Fig. 9 Effect of multipath time delay on detection 
In Fig. 10, we demonstrate the sensitivity of the multipath detection algorithm to the spatial separation between the multipath and direct signal AOAs. Fig. 10 was generated from a Monte-Carlo simulation involving two signals: one with the direct GPS and a single multipath signal and the other with just the direct GPS signal. These two signals represent the two signals under our two competing hypothesis. A grid search is performed over the plot in Fig. 10: the direct signal's AOA is varied from zero to ninety degrees in five degree increments and at direct signal AOA, the multipath AOA is varied from the direct signal AOA by zero to 15 degrees (in 1 degree increments). For each effective $\left(\gamma_{0}, \gamma_{1}\right)$ pair, 2,500 Monte-Carlo simulations are run. The empirical distribution functions for both signals are computed and the critical value at which the estimated type I and type II errors are the same is found. This approach gives us an estimate for the detection errors without weighting a specific detection error over the other. The value of $\alpha$ (or equivalently $\beta$ ), is then chosen as our detection performance metric and are the contour values plotted in Fig. 10.

Fig. 10 shows that the spatial proximity of the multipath signal has a great effect on the detection performance when direct signal AOA is relatively small. For example, when $\gamma_{0}<20^{\circ}$, the multipath AOA has to be at least $12^{\circ}$ greater than the direct signal's to ensure that both $\alpha$ and $\beta$ are less than 20\%. As the desired LOS AOA increases however, the two signals can get relatively close to each other before performance is significantly degraded. When $\gamma_{0}=60^{\circ}$, the multipath signal AOA can be about $5^{\circ}$ away from the direct signal AOA and still be detected with less than $5 \%$ false alarm and miss detection rate.

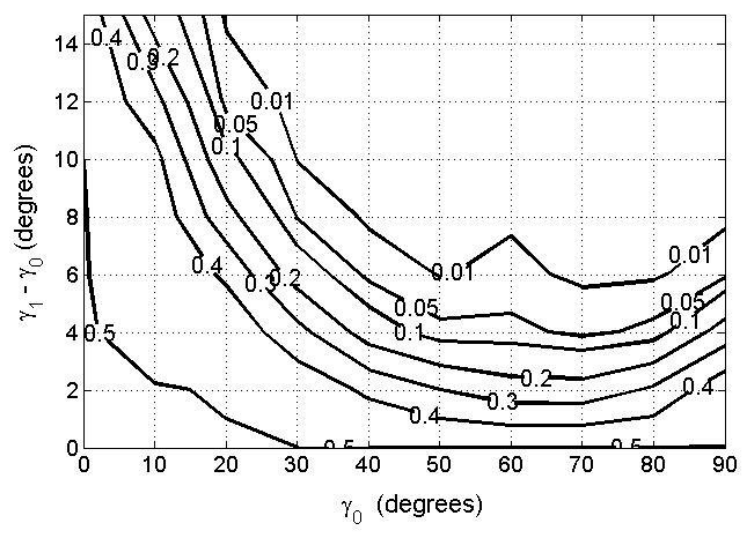

Fig. 10 Effect of relative multipath and direct signal AOA on detection.

\section{CONCLUSIONS}

Multipath is one of the major error sources in high accuracy GPS applications. The most difficult type of multipath are those whose time delay relative to the direct GPS signal, is short. For these types of multipath, existing methods to may not be able to detect the presence of multipath and therefore not recognize the error produced by the multipath. The ANOVA algorithm presented in paper takes advantage of the spatial diversity between the multipath and the direct GPS signal to detect the presence of such multipath.

We have shown that the ANOVA-based algorithm can detect the presence of multipath using 3-10 CA code periods with modest computational cost. The algorithm requires the construction of a single weight vector based on known direct signal AOA and multiplication of the weight vector to the correlator outputs. The ANOVA-based algorithm compliments previous methods, in that its performance improves as the multipath time delay decreases. As the angle of arrival for the multipath and the direct signal becomes close to each other, the detection performance is degraded as expected. Simulations suggest however that this reduction in performance is limited to a relatively small region and that by increasing the number of array elements, performance can be improved to the desired level.

The goal of this paper was to show that multipath signal detection for short time delay multipath can be performed using the spatial diversity between the desired and undesired signal in a way that was generally applicable, simple to implement, and yet had good performance. Improvements of this method are planned considering the optimization of the detection method by comparing the performance of the ANOVA method with various eigenstructure techniques. The incorporation of the temporal and spatial diversity should help also improve detection and make the method more robust.

\section{BIBLIOGRAPGHY}

[1] Dierendonck, A. J., P. Fenton, and T. Ford, "Theory and Performance of Narrow Correlator Spacing in a GPS Receiver", NAVIGATION, Journal of the Institute of Navigation, vol. 39, no. 3, pp. 265-283, 1992.

[2] Garin, L. and J.M. Rousseau, “Enhanced Strobe Correlator Multipath Mitigation for Carrier Code" , Proc $10^{\text {th }}$ International Technical Meeting of the Satellite Division of the Institute of Navigation, ION-GPS, vol. 1, pp. 559-568, 1997.

[3] Townsend, B. and P. Fenton, “A Practical Approach to the Reduction of Pseudorange Multipath Errors in a L1 GPS Receiver", Proceedings of the $7^{\text {th }}$ International Technical Meeting of the Satellite Division of the Institute of Navigation, Part 1 (of 2), Proceedings of ION GPS, vol. 1, pp. 143-148, 1994. 
This paper will appear in Proc. 2008 Joint IEEE PLANs and ION Annual Meeting

[4] R.D.J. van Nee, “The Multipath Estimating Delay Lock Loop", IEEE $2^{\text {nd }}$ International Symposium on Spread Spectrum Techniques and Applications, Yokohama, Japan, pp. 39-42, 1992.

[5] Cahn, C. and M. M. Chansarkar, "Multipath Corrections for a GPS Receiver", Proceedings of the $10^{\text {th }}$ International Technical Meeting of the Satellite Division of the Institute of Navigation, ION-GPS, vol. 1, pp. 551-557, 1997.

[6] L.R. Weil, "Multipath Mitigation using Modernized GPS Signals: How Good Can it Get?", Proceedings of the $15 \$ \wedge$ th $\} \$$ International Technical Meeting of the Satellite Division of the Institute of Navigation ION GPS, pp. 493 505, 2002.

[7] Selva, J., "Efficient Multipath Mitigation in Navigation Systsems", Ph.D. Thesis, Universitat Politècnica de Catalunya, 2003.

[8] Sahmoudi, M. and M.G. Amin, "Fast Iterative MaximumLikelihood Algorithm (FILMA) for Multipath Mitigation in Next Generation of GNSS Receivers", $40^{\text {th }}$ Asilomar Conference on Signals, Systems and Computers, 2006. ACSSC '06, pp. 579 - 584, 2006.

[9] Soubielle, J., I. Fijalkow, P. Duvaut, and A. Bibaut, “GPS Positioning in a Multipath Environment", IEEE Trans Signal Proc., vol. 50, no. 1, pp. 141 - 150, 2002.

[10] Casella, G. and R.L. Berger, "Statistical Inference", Duxbury Thomson Learning, $2^{\text {nd }}$ edition, 2002, Chapter 11.

[11] Misra, P. and P. Enge, "Global Positioning System: Signals, Measurements, and Performance ", Ganga-Jamuna Press, $2^{\text {nd }}$ edition, 2006, Chapter 11.

[12] Cohen, J. "Statistical Power Analysis for the Behavioral Sciences", Lawrence Erlbaum, $2^{\text {nd }}$ edition, 1988, Chapters 2 and 3. 